\title{
At the Tip of an MeV Beam: Provoking Cells and Performing Tomographic Imaging
}

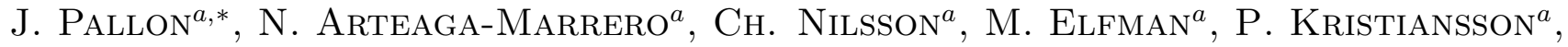

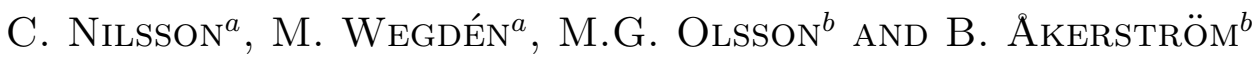 \\ ${ }^{a}$ Division of Nuclear Physics, Physics Department, Lund University \\ Professorsgatan 1, P.O. Box 118, 22100 Lund, Sweden \\ ${ }^{b}$ Clinical and Experimental Infection Medicine (BMC) \\ Sölvegatan 19, B14, SE-221 84, Lund, Sweden
}

\begin{abstract}
Biological applications of ion beams have recently become a new important research field using single ion hit facilities to study individual living cells and their response to the hit of a counted number of ions. One motivation is the search for a better understanding of the fundamental processes taking place in cells and organs as a result of irradiation. Another comes from the increasing interest in using high energy protons and heavy ions as a modality for radiotherapy of deep seated tumours. In the view of treatment efficiency, study of cell culture behaviour under controlled radiation experiments, and in different chemical environments at single ion hit facilities, is a first step towards a better understanding of the processes. Tomographic techniques are applicable to situations where you need information of the inside of an object but do not want to section it into thin slices or cannot do it. Using focused $\mathrm{MeV}$ ion beams for tomography restricts the sample size to the order of 10-100 $\mu \mathrm{m}$, depending of the initial energy. On the other hand, the ability to focus at a sub-micrometer level makes ion beams well suited for analyses of small sized objects as cells, spores, etc. The scanning transmission ion microscopy mode of tomography gives the mass density and corresponding morphological structure of holes and pores. It can then be used to correct the results from the other mode, particle induced X-ray emission tomography. Here is discussed a porosity analysis of bentonite clay that is planned to form an important buffer zone around canisters filled with spent nuclear reactor fuel waste deposited $500 \mathrm{~m}$ underground in Sweden.
\end{abstract}

PACS numbers: 07.79.--v, 29.40.Wk, 87.53.-j, 87.53.Ay, 25.40.Cm, 29.30.Ep, 29.30.Kv, 61.43.Gt

\section{Background}

\subsection{Development of irradiation facilities}

During the recent years there has been a European move towards developing high resolution ion beam facilities to be used in the study of cellular responses to single ion hits $[1,2]$. Those systems utilise the counted, localised and focused $\mathrm{MeV}$ ions to interact with monolayer biological experimental systems to induce localised, quantified damage with the purpose to study the biological effects originating from the interaction. It has since more than a decade been a research field in the borderland between physics and biology [3, 4] and today a considerable number of single ion hit facilities have been developed worldwide (see e.g. $[5,6]$ ). A single ion hit facility (SIHF) is a modified nuclear microprobe specially adopted for the task of shooting single, positioned ions [7].

Many experiments focus on the effects of low dose irradiation: bystander effect, genomic instability and adaptive responses [8]. There are several phenomena that

* corresponding author; e-mail: Jan.Pallon@nuclear.lu.se are not well understood, e.g. the limits for double-strain breaks, cell-to-cell communication and interactions that indicate both increased sensitivity versus radiation as well as increased protection against radiation (adaptative response). This type of experiments can hardly be done without the use of well controlled ion exposures (local energy transfer to the cell, number of ion hits per cell and localization within the cell).

\subsection{Relation to proton therapy}

In radiation therapy there is always a delicate balance between delivering high enough dose to the tumour and at the same time low enough to minimize the damage to surrounding tissues. Proton therapy, where high energy protons are used, has the physical advantage over photon therapy that the ions deliver most of their energy at the end of the path (the stopping power is low and constant up to the end where it increases in the Bragg peak), and are thus favourable for treating deep lying tumours in the body. It is not well known how tissue cells that are near the border of the irradiation volume (the Bragg peak) react as there is a sharp gradient in deposited energy/mass element going outwards into healthy tissue. 


\section{The single ion hit facility}

A SIHF is a combination of an accelerator based microprobe system and an irradiation chamber that allows single $\mathrm{MeV}$ ions to be extracted through a thin vacuum window into air, pass a second window to hit single living cells kept in a nutrient solution. The cells are grown in culture and normally attached to this second window. The passage of each ion is detected and is used to trigger a fast beam blanking system that sweeps off the beam and allows each cell to be hit by just one ion. Through collimation, the particle flux is limited to less than 1000 ions/s. A fast (160 ns) blanking system, triggered by an ion hit detector will block the beam [9] to prevent unwanted ions to reach the exposure area, see Fig. 1. By (magnetic) steering the beam is then directed to hit a new cell and thus a precise dose can be given each cell (1, 2, etc. ions). At least 10000 cells/hour can be exposed using this technique. After irradiation, the cell culture is moved to an incubator where cell vitality is monitored and followed up in different ways.

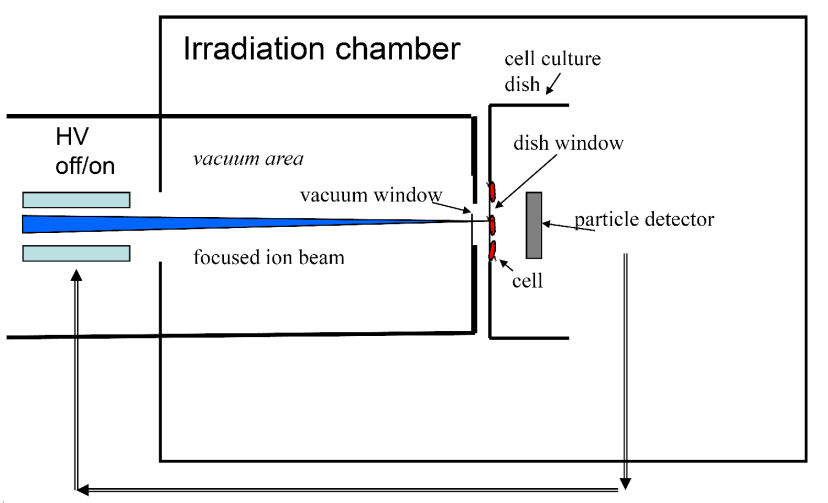

Fig. 1. Principle of a system for irradiation of individual cells using single ions. The high energy ions are passing a thin window into atmospheric condition and a second window to hit the cells staying in culture media. A particle detector behind the cells serves as a hit indicator. As an alternative (not shown), a pre-cell detector can be placed in the vacuum area. The hit (after logical operations) triggers the HV-beam deflector.

The first SIHF at Gray lab and Columbia had collimated beams using capillary exits [10] and a mechanical stage to move the cells. Typically $2-5$ micrometers beam diameter was achieved and much care needed to reduce unavoidable slit scattering.

However, it is advantageous to have the SIHF based on focusing as it permits sub-micrometer precision and detailed sub-cellular information. For cellular irradiation response studies it is essential to know where the ion track was created in a cell - e.g. crossing the nucleus or passing the cytoplasm. In addition to the higher precision, an advantage with a focused beam is that with a beam scanning device cell selection can be made much faster than using mechanical scanning; and in addition with better precision in position. Thus a higher number of cells can be exposed with a larger accuracy allowing more investigations to be done in each irradiation experiment.

\subsection{Vertical cell dish}

If the ion beam is coming upwards (or downwards), a simple horizontal cell dish arrangement can be used. Most microprobes using focusing lenses are however constructed for a horizontal beam and thus the cell culture must be positioned vertically. There are no indications that the cells should fall off, once attached to the cell dish window they will stay. One complication is to assure that the cell culture medium surrounds the cells enough during irradiation to keep them healthy. From physical reasons, there will always be a thin layer due to capillary forces covering the cells until evaporation causes a drying out. This safe time window depends on the actual setup; temperature, humidity, cell type, etc. and must be found experimentally.

\subsection{Limits to high resolution}

While in vacuum there is a full control of beam size and position, but at the point where the ions pass through the exit window and enters the atmospheric conditions their energy and direction becomes a function of the material they traverse. In general terms, the ions will suffer energy loss, energy straggling and lateral straggling. Of those the lateral straggling is the most limiting factor when it comes to aiming at specific cell structures, and the spreading increases with the distance from the first non-vacuum scattering point. Thus to minimise the beam spot, the cell dish should ideally coincide with the exit window. In reality there must be a separate exit window and cell dish window and the task in practice is to minimise the air gap in between to be typically less than $0.1 \mathrm{~mm}$. To minimise the initial scattering at the exit window, as thin and strong material as possible should be chosen. A common material used is $\mathrm{Si}_{3} \mathrm{~N}_{4}$, commercially available with windows having a diameter of about $3 \mathrm{~mm}$ and a thickness 100-200 $\mathrm{nm}$.

Designing the SIHF for protons is the most demanding task as heavier ions (alpha-particles, carbon ions, etc.) scatter less than protons. Also when it comes to pre-cell hit-detection, see below, protons are the most difficult case to handle.

Because of energy loss, the ions will have a given range in matter, and the value of the initial ion energy determines how thick cell layer and culture medium that can be traversed. For the case of post-cell hit detection, the culture medium surrounding the cells must be minimised to a point where the bombarding ions safely can be detected after the passage of windows, cells and medium. This is an important limitation for many microprobes working in the $2-3 \mathrm{MeV}$ range. As an example, a $100 \mu \mathrm{m}$ layer needs energies from $2.5 \mathrm{MeV}$ protons and up; a $75 \mu \mathrm{m}$ layer can be passed from $2 \mathrm{MeV}$ and above; while $50 \mu \mathrm{m}$ allows $1.7 \mathrm{MeV}$ protons to be used. Thus it is very important to improve the possibility to hold a thin and 
homogeneous liquid layer in place, e.g. by introducing microstructures produced by lithography.

\subsection{Microscopy, cell recognition and positioning}

Experiments with SIHF require irradiation of hundreds or thousands of cells with precisely the same number of ion traversals through all cells. Cells are grown in culture media and will attach to the bottom of a dish. Before irradiation, the location of each cell and its nucleus must be determined and registered using suitable software, and the corresponding coordinates then are used to precisely deflect the single ions to hit just the selected position within the cell. To enhance optical contrast, imaging using fluorescence additives to the cells can be used. Fluorescence imaging can be highly specific, has a good signal-to-noise ratio and is suitable for routine work and automatic cell identification. However, there are potential cell toxicity problems. Non-fluorescence imaging is non-toxic for the cells, but much more complicated. It relies on techniques like dark-field/phase contrast methods, contrast generation from optical path differences or interferometric methods. Advanced imaging and cell finding software is necessary.

\subsection{Pre-cell detection}

At irradiation, each single ion needs to be detected to control the beam deflection system and thus the applied dose to the cells. The simplest and most straightforward solution is to use post-cell detection, often semiconductor detectors, e.g. Cracow [11], LIPSION [12] and SNAKE [13]. However, as discussed above, it poses limitation both in terms of cell layer thickness and microscopy, and the alternative is pre-cell detection, in the vacuum region just in front of the exit foil. Setups with pre-cell detectors, using thin plastic scintillation detectors or secondary electrons detected in e.g. a channeltron, are described at CENBG [14], GCI [15], PTB [16]. An exit window of $100 \mathrm{~nm} \mathrm{Si}_{3} \mathrm{~N}_{4}$ or thin boron doped diamond films [17] can serve both as window and scintillator (if covered with thin CsI generates secondary electrons). Detection efficiency of $99.5 \%$ was reported for carbon ions [18], but the corresponding figure for protons was lower [19]. Recent results from the Kraków group [20] indicates that the $\mathrm{Si}_{3} \mathrm{~N}_{4}$ membrane efficiency for proton registration was only $10 \%$, while for $\mathrm{Si}_{3} \mathrm{~N}_{4}$ covered with CsI the best result achieved was $61 \%$. Efficiency well above $90 \%$ is vital for the technique.

Another approach has been taken in Lund. Thin (10 $14 \mu \mathrm{m}$ ) silicon $E$-detectors developed for high energy physics telescopes have been tested as pre-cell detector candidates. The best results were obtained using a $2 \times 2 \mathrm{~mm}^{2}$ detector, having an acceptable signal to noise level [21]. Using a new version, $1 \times 1 \mathrm{~mm}^{2}$ and less than $9 \mu \mathrm{m}$ thick detector yielded efficiency values close to $100 \%$ for proton energies up to $2.2 \mathrm{MeV}$ [22], which is very promising.

The drawback with the $E$ detector is that it causes more lateral scattering that limits the lateral precision at irradiation and excludes studies aiming at the most detailed sub-cellular components. As a conclusion, it is the biological task that at the end sets the limits to what type of hit detection that can be used.

\section{Responses to radiation}

As already mentioned, there is increasing interest in the role of "non-targeted" effects where cells respond indirectly to energy deposited by radiations. One such effect is the occurrence of damage arising from radiation induced cell signalling both intracellular (i.e. from the cytoplasm to the nucleus) and inter-cellular (from cell to cell). The interest in inter-cellular signalling followed on reports by Nagasawa and Little [23], Deshpande et al. [24] and Hickman et al. [25]. The implication of these findings is that damage is occurring in non-irradiated cells in response to signals from neighbouring irradiated cells. This phenomenon has been termed the "bystander effect" and microbeam techniques that allow the selective irradiation of cells (or even just a single cell) with micron or submicron targeting capability within a population are clearly advantageous for this type of study.

The genotoxic effect of charged particle radiation on living cells is a result of interactions among biological matter; primary particles traversing the cell, positive ions and secondary electrons produced along the track of charged particles and of chemical reactions with free radicals or other reactive oxygen species created by radiolysis of water in the cell. Those interactions can be tracked down to a level of a single chromosome or even of a single DNA strand. The development of SIHFs allows achieving a better linear energy transfer (LET) definition, greater range of LET using heavy ion beams, and higher aiming accuracy.

\subsection{Mechanisms of cell death and the bystander effect}

In tumour therapy, the desired effects of proton irradiation involve cell death. The findings of new biological responses to proton-irradiation, such as membrane-mediated signalling and the bystander effect have stimulated a wave of new research on mechanisms of cell death. Bystander effects are defined as secondary effects on cells not directly hit by the irradiation particles. Thus, our understanding of the mechanisms of proton irradiation-induced cell death has undergone a change from a view locked into the notion of direct DNA damage and repair to one that encompasses the important intracellular and intercellular signalling pathways present at the cell and tissue level (see [26] for a review). According to the present paradigm, cell death is either accidental, necrosis, or programmed, apoptosis and it can be expected that proton beam irradiation induces both necrosis and apoptosis. However, many mechanisms exist that aim to rescue cells after radiation damage, and two of the most important of these are cell-cycle arrest and DNA-repair. A major molecular regulator of the response to various stress factors, including radiation, is the small protein 
p21 [27]. This protein is up regulated in response to oxidation, UV-radiation, etc., and induce G1 cell-cycle arrest, DNA-repair and inhibit apoptosis.

We hypothesise that proton irradiation induces primary oxidative stress by ionisation of solvent and cell components directly hit by the proton beam, and secondary oxidative stress by proton-induced cell necrosis. Oxidative stress is defined as increased tissue oxidation, due to imbalance between generation of oxidative compounds and detoxification of oxidants/repair of oxidated tissue (reviewed in [28]). The major mediators of oxidative stress are reactive oxygen species (ROS) which include hydrogen peroxide, superoxide and the hydroxyl radical. ROS, oxidants, and free radicals react with proteins, DNA and other molecular components to cause oxidation of human cells and tissues, which can lead to unwanted modifications of the target molecules, loss of function and cell death. Necrosis induces oxidative stress mainly by disruption of the compartmentalisation of oxidative processes. The research in the Lund group has accordingly been focused on detecting ROS effects invoked by the irradiation using e.g. the fluorescent oxidation-sensitive probe H2DCFDA. Typically, following irradiation, signs of oxidation start to develop after three hours. A large amount of bystander cells become damaged due to oxidation and a few hours later, the cells start to die. Presently the studies have a more qualitative character but we plan to characterise cell death and oxidation temporally and spatially and also investigate the importance of the medium for the induction of cell death by culturing non-irradiated cells in the irradiated medium.

\subsection{Concluding remarks}

In the perspective of coming facilities for proton therapy, experiments at SIHF are useful to build procedures for controlled laboratory cell radiations where corresponding results are applicable to future clinical situations. It allows model experiments to be carried out where effects are studied on scales from molecular, cellular and thin tissue layers. The SIHF will be an extremely useful tool for investigating issues related to proton/alpha particle radiobiology where findings of the role of oxidants and antioxidants may be used to maximise the therapeutic effect while minimising unwanted damage. In addition, the studies contribute to fundamental understanding of radiation induced cell damage mechanisms.

\section{Tomography}

Today, tomography is used in many research areas and in industry to perform non-destructive examinations and gain true three-dimensional information on various objects. Not only are X-rays used but also photons in general and also particle irradiation. The range in object size stretches from proteins imaged by NMR/MRI to investigations of the inside of the Earth by seismic tomography.

Two-dimensional mapping with ion microprobes was extended to tomographic imaging by several research groups $[29,30]$. By using the full 3D information small structures inside samples can be imaged, e.g. cellular structures or porosity. Tomographic investigations require very small samples, are time consuming and have therefore not replaced the standard two-dimensional mapping that delivers sufficient information in most applications. For specific questions, however, tomography can be a source of valuable information, which simplifies the interpretation of data and makes features visible, which cannot be distinguished in two dimensions.

The principle of computed tomography is combination of "slicing" and "writing". An image of the slice can be made mathematically by irradiating the object in many different angles and measuring how the radiation is affected by the passage through the object, either in terms of energy loss or attenuation. Data on transmitted energy or intensity for a specific angle is called a projection and represents the line integral of the measured parameter. By combining a set of projections from typically 180 $360^{\circ}$ around the object, a mathematical reconstruction can be made. By stacking many such reconstructed cross-sections on top of each other, a full three-dimensional image of the object can be done.

\subsection{Ion beam tomography}

Ions interact differently with matter than X-rays, as they are both slowed down and scattered when traversing matter. This limits the useful range for $\mathrm{MeV}$ ions to $10-100 \mu \mathrm{m}$, depending on the initial energy, ion type and target material. The ion range limits the dimensions on the sample that are to be analysed. On the other hand, the ability to focus the beam to sub-micrometer size and the very low lateral straggling compared to e.g. electrons makes high resolution ion beam tomography very attractive to study microscopic structures like cells, spores, pores etc [31].

For the case of ion beam tomography, there are two types developed, scanning transmission ion microscopy (STIM), tomography based on the mass density, and PIXE tomography based on the elemental distribution. The mass density data is useful to image the morphology in the sample and reveal internal structures or pores. Three-dimensional density information can then help to perform a better mass normalisation for the PIXE tomography experiments.

\subsection{A feasibility study for porosity characterisation of bentonite clay}

Bentonite clay is planned to be used in the KBS-3 $[31,32]$ concept for a future nuclear high level waste repository in Sweden. In the concept the spent nuclear fuel is encapsulated in a copper canister using friction stir welding. The copper canister is embedded in compacted bentonite and deposited at $500 \mathrm{~m}$ depth in granite bedrock. The compacted bentonite will act as a buffer material, giving mechanical support for the copper canister, reducing water movements and capturing potentially escaping radio-nuclides. 
Bentonite is a natural clay, which has a high swelling capacity in water, originating from its high content of smectite minerals. Smectite minerals, with montmorillonite as the most common, are sheet silicates, which can intercalate water into their structure by hydrating interlayer cations. This causes expansion of the interlayer distances and an exceptional swelling capacity, which makes bentonite a suitable buffer material that works as a sealant and barrier [33]. The hydration process is reversible, and in a dry atmosphere or during heating, both water and hydrated cations can leave the structure of montmorillonite [34].

The final pore size distribution determines the hydraulic permeability and governs the possible transport of colloids through the clay. Heterogeneity in the material, compaction and swelling may result in porosity, both on the nano- and micrometer scale, which may affect the permeability of the clay and may mediate the transport of radio-nuclides, cations and corrosion products.

In addition to STIM, PIXE and p-p (hydrogen) analyses performed on thin slices of bentonite, STIM tomography was also made to find out if the threedimensional mass density structure obtained would be detailed enough to examine micrometer-sized pore distributions.

The sample preparation in this case was non-trivial, the range of protons in bentonite was estimated to be $70 \mu \mathrm{m}$. The clay is brittle and the ideal situation would be to have the clay enclosed in a glass micro-capillary. However, no such were found commercially available and in addition to other drawbacks when using a surrounding glass material, a simpler procedure was chosen. A small amount of the bentonite clay was dispersed in milliQ water and then allowed to dry on a Mylar foil film. After 24-48 hours, the Mylar foil could be removed and a thin, self supporting layer of clay resulted. From this, strips were cut and mounted on the rotating needle tip used for tomography.

For a full data set of STIM tomography, the projection maps must be acquired at equidistant angles covering $0-180^{\circ}$. In the determination of the residual energy, the average value of a number of transmitted ions was used, since this is known to be better in resolving small structures than the median value [35] and can thus better resolve structures and pores in the bentonite. Typically 40 protons were detected per pixel which was sufficient for good energy determination, however, data acquisition at every projection angle turned out to be very time demanding.

After data collection, the energy loss was converted to mass density and a standard filtered backprojection algorithm [36] was applied to reconstruct the data. An iso-density surface is shown in Fig. 2a to illustrate the morphology of the bentonite strip. Here the data were smoothed and a threshold of $0.6 \mathrm{~g} / \mathrm{cm}^{3}$ was set as the iso-density value to be shown. The internal structure of the sample can be further investigated in Fig. $2 \mathrm{~b}$ where 30 reconstructed cross-sections have been stacked and a vol-
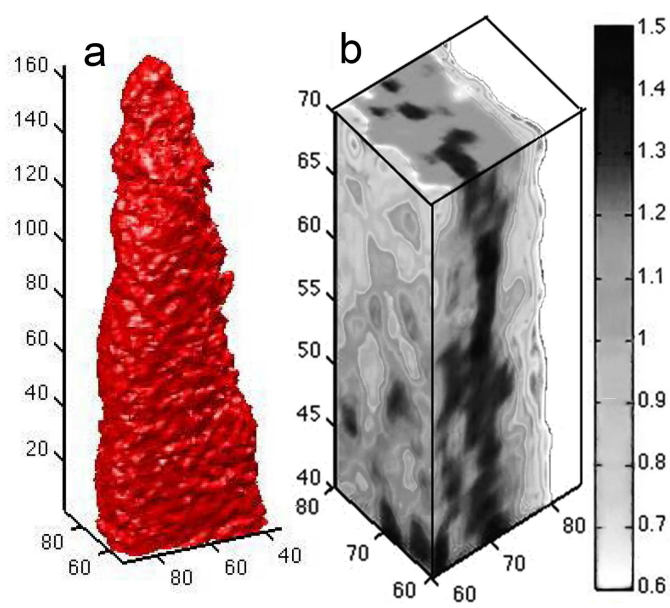

Fig. 2. (a) An iso-density surface is shown to illustrate the morphology of the bentonite strip. Here the data were smoothed and a threshold of $0.6 \mathrm{~g} / \mathrm{cm}^{3}$ was set as the iso-density value to be shown. (b) The internal structure of the sample from 30 reconstructed crosssections that are stacked and a volume $(12 \mu \mathrm{m} \times 12 \mu \mathrm{m} \times$ $18 \mu \mathrm{m})$ is cut out. Although the number of projections was fewer than for an ideal reconstruction a clear internal structure can be discerned with regions of higher mass density, possibly corresponding to accessory minerals, and regions with lower density which might indicate micrometer-sized pores.

ume $(12 \mu \mathrm{m} \times 12 \mu \mathrm{m} \times 18 \mu \mathrm{m})$ has been cut out. Although the number of projections was fewer than for an ideal reconstruction, a clear internal structure can be discerned with regions of higher mass density, possibly corresponding to accessory minerals, and regions with lower density which might indicate micrometer-sized pores. The mass density in the pores is not zero as could be expected, and is an effect of the reconstruction technique, where data is smeared out in the backprojection process. This effect is in consistence with simulated reconstructions of noise-free phantom projection data performed with the filtered backprojection technique at the ideal number of projection angles. Small pores, with a size comparable to the pixel width will not be reconstructed as zero-density values, but the agreement increases for larger pores. It must also be emphasised that proper alignment of the rotation axis is crucial for the quality of the reconstructed image, but it is also very difficult to achieve experimentally.

\subsection{Conclusions}

STIM tomography was successfully applied to analysis of bentonite strips, and yielded information on the internal structure and porosity on a micrometer scale. However, the long beam time needed for data acquisition makes STIM tomography less suitable for standard porosity studies. The sample preparation technique must be further developed to be able to preserve the natural structure of the clay. Another goal is to repeat the study having different water content in the clay. In this case, 
however, the $\mathrm{p}-\mathrm{p}$ coincidence technique applied to thin bentonite films could be a powerful alternative.

\section{References}

[1] M. Folkard, B. Vojnovic, K.M. Prise, B.D. Michael, Nucl. Instrum. Methods Phys. Res. B 188, 49 (2002).

[2] M. Folkard, B. Vojnovic, S. Gilchrist, K.M. Prise, B.D. Michael, Nucl. Instrum. Methods Phys. Res. B 210, 302 (2003).

[3] M. Folkard, B. Vojnovic, K.M. Prise, A.G. Bowey, R.J. Locke, G. Schettino, B.D. Michael, Int. J. Radiat. Biol. 72, 375 (1997).

[4] M. Folkard, B. Vojnovic, K.J. Hollis, A.G. Bowey, S.J. Watts, G. Schettino, K.M. Prise, B.D. Michael, Int. J. Radiat. Biol. 72, 387 (1997).

[5] M. Heiss, B.E. Fischer, B. Jakob, C. Fournier, G. Becker, G. Taucher-Scholz, Radiat. Res. 165, 231 (2006).

[6] A.W. Bigelow, G.J. Ross, G. Randers-Pehrson, D.J. Brenner, Nucl. Instrum. Methods Phys. Res. B 231, 202 (2005).

[7] B.E. Fischer, M. Heiss, M. Cholewa, Nucl. Instrum. Methods Phys. Res. B 210, 285 (2003).

[8] M.A. Kadhim, S.R. Moore, E.H. Goodwin, Mutation Res. 568, 21 (2004).

[9] V. Auzelyte, M. Elfman, P. Kristiansson, K. Malmqvist, L. Wallman, Ch. Nilsson, J. Pallon, A. Shariff, M. Wegdén, Nucl. Instrum. Methods Phys. Res. B 219-220, 485 (2004).

[10] M. Folkard, B. Vojnovic, K.M. Prise, A.G. Bowey, G. Schettino, B.D. Michael, Int. J. Radiat. Biol. 72, 387 (1997).

[11] W. Polak, O. Veselov, J. Lekki, Z. Stachura, M. Zazula, R. Ugenskiene, M. Polak, J. Styczen, Nucl. Instrum. Methods Phys. Res. B 249, 743 (2006).

[12] T. Reinert, A. Fiedler, J. Skopek, J. Tanner, J. Vogt, T. Butz, Nuclear Instrum. Methods Phys. Res. B 219-220, 77 (2004).

[13] A. Hauptner, S. Dietzel, G.A. Drexler, P. Reichart, R. Krücken, T. Cremer, A.A. Freidl, G. Dollinger, Radiat. Environm. Biophys. 42, 237 (2005).

[14] Ph. Barberet, A. Balana, S. Incerti, C. Michelet-Habchi, Ph. Moretto, Th. Pouthier, Rev. Sci. Instrum. 76, 015101 (2004).

[15] M. Folkard, B. Vojnovic, S. Gilchrist, K.M. Prise, B.D. Michael, Nucl. Instrum. Methods Phys. Res. B 210, 302 (2003).

[16] K.D. Greif, H.J. Brede, D. Frankenberg, U. Giesen, Nucl. Instrum. Methods Phys. Res. B 217, 505 (2004).

[17] T. Kamiya, M. Cholewa, A. Saint, S. Prawer, G.J.F. Legge, J.E. Butler, D.J. Vestyck, Appl. Phys. Lett. 71, 1875 (1997).
[18] M. Cholewa, B.E. Fischer, M. Heiß, Nucl. Instrum. Methods Phys. Res. B 210, 296 (2003).

[19] W. Polak, J. Lekki, J. Grybos, R. Hajduk, M. Cholewa, O. Kukharenko, Z. Stachura, Nukleonika 48, 25 (2003).

[20] A.W. Polak, R. Hajduk, S. Lebed, J. Lekki, T. Horwacik, S. Maranda, T. Pieprzyca, C. Sarnecki, Z. Stachura, Z. Szklarz, O. Veselov, J. Styczeń, IFJ Report No. 1955/AP, Kraków 2004.

[21] Ch. Nilsson, J. Pallon, G. Thungström, N. Arteaga, V. Auzelyte, M. Elfman, P. Kristiansson, C. Nilsson, M. Wegdén, Nucl. Instrum. Methods Phys. Res. B 249, 924 (2006).

[22] Ch. Nilsson, J. Pallon, G. Thungström, N. Arteaga Marrero, M. Elfman, P. Kristiansson, C. Nilsson, M. Wegdén, Nucl. Instrum. Methods Phys. Res. B 266, 4808 (2008).

[23] H. Nagasawa, J.B. Little, Cancer Res. 52, 6394 (1992).

[24] A. Deshpande, E.H. Goodwin, S.M. Bailey, B. Marrone, B. Lehnert, Radiat. Res. 145, 260 (1996).

[25] A.W. Hickman, R.J. Jaramillo, J.F. Lechner, N.F. Johnson, Cancer Res. 54, 5797 (1994).

[26] K.M. Prise, G. Schettino, M. Folkard, K.D. Held, Lancet Oncol. 6, 520 (2005).

[27] M.A. O'Reilly, Antiox. Red Signal 7, 108 (2005).

[28] Free Radicals in Biology and Medicine, Eds. B. Halliwell, J.M.C. Gutteridge, Oxford University Press, Oxford 2002.

[29] J. Huddleston, I.G. Hutchinsson, T.P. Pierce, J. Foster, Nucl. Instrum. Methods Phys. Res. B 3, 584 (1984).

[30] M. Cholewa, Yang Ping, Ng May Ling, Li Zhi Juan, H.O. Mosert, Teukuang Hwu, T.E. Guruyev, Nucl. Instrum. Methods 197, 157 (1982).

[31] M. Wegdén, Ph.D. Thesis, Department of Physics, Lund University, 2007, LUTFD2/(TFKF-1035)/1$65 /(2007)$.

[32] SKB Report, R-98-10, SKB, Swedish Nuclear Fuel and Waste Management Co., 1998.

[33] O. Karnland, M. Birgersson, Technical report, TR06-11, SKB, Swedish Nuclear Fuel and Waste Management Co., 2006.

[34] H.-R. Wenk, A. Bulakh, Minerals: Their Constitution and Origin, Cambridge University Press, Cambridge 2004.

[35] G.S. Bench, G.J.F. Legge, Nucl. Instrum. Methods B 40/41, 655 (1989).

[36] A.C. Kak, M. Slaney, Principles of Computerized Tomography Imaging, IEEE Press, New York 1988. 